\title{
Hypervirulent Klebsiella pneumoniae carriage in polyclinic attendees and national servicemen presenting with diarrhoea
}

\section{Dear Editor,}

Klebsiella pneumoniae liver abscess is an invasive syndrome that mainly affects people living in East Asia. It especially affects adults with diabetes and is caused by hypervirulent strains that possess the rmpA gene (regulator of mucoid phenotype A), ironsequestering genes, and usually belong to capsule types $\mathrm{K} 1$ and K2. K. pneumoniae liver abscesses may arise from intestinal colonisation with hypervirulent strains; however, the prevalence of carriage of these strains in the local population in Singapore is not welldescribed. We were also interested to see if ethnicity (possibly because of dietary preferences) has an influence on the carriage of such strains. Two outpatient populations were studied in 2013-2014: patients attending polyclinic for faecal occult blood testing, and national servicemen (Singapore and permanent resident men 18 years and above who are liable to join the national defence force) presenting with diarrhoea that had stool sent for bacterial and parasite investigation.

Residual stool left over from faecal occult blood testing, and bacterial and parasite investigation was screened for $K$. pneumoniae using Klebsiella selective agar. Any suspect $K$. pneumoniae colonies were identified by matrix-associated laser desorption/ionisationtime of flight mass spectrometry, and tested for antimicrobial susceptibility (ampicillin, amoxicillin- clavulanate, piperacillin-tazobactam, cephalothin, ceftriaxone, cefepime, aztreonam, gentamicin, amikacin, ciprofloxacin, ertapenem and meropenem), and virulence and capsule genes by multiplex polymerase chain reaction. ${ }^{1}$ A total of 438 stool samples were tested from patients (207 men and 231 women) in the Singapore polyclinics. The ages of the patients ranged from 3-95 years with a mean of 60 years. The ethnic breakdown of patients sending stool samples from polyclinics was: Chinese $(374,85.4 \%$, ), Malay $(31,7.1 \%)$, Indian (17, $3.9 \%)$, and others $(16,3.7 \%)$. Of these, 153 stools had $K$. pneumoniae isolated, and 36 isolates had the rmpA gene. The capsule types of rmpA positive strains are summarised in Table 1: 14 had capsule K1 (9 men and 5 women, age range 44-82 years, mean 63 years) and 8 had capsule K2 ( 7 men and 1 woman, age range 42-72 years, mean 60 years). A total of 618 stool samples were tested from national servicemen. The ethnic breakdown of national servicemen sending stool samples was: Chinese (448, 72.5\%), Malay $(116,18.8 \%)$, Indian $(44,7.1 \%)$, and others $(10,1.6 \%)$. Of these, 173 stools had $K$. pneumoniae isolated and 19 isolates had the rmpA gene. Of the latter, 14 had capsule $\mathrm{K} 1$ and 3 had capsule $\mathrm{K} 2$.

All K. pneumoniae strains were susceptible to multiple antimicrobials. The number of rmpA positive isolates was too small to pick up any clustering of capsule serotype by ethnicity.

Table 1. Capsule types of rmpA-positive Klebsiella pneumoniae isolated from patients by ethnicity

\begin{tabular}{|c|c|c|c|c|c|c|c|c|}
\hline & \multicolumn{8}{|c|}{ Capsule types } \\
\hline & K1 & K2 & K5 & K20 & K54 & K57 & Others & Total \\
\hline \multicolumn{9}{|c|}{ Ethnicity (polyclinic) } \\
\hline Chinese & 13 & 7 & 1 & 2 & 1 & 4 & 2 & 30 \\
\hline Malay & & & & 1 & & 1 & & 2 \\
\hline Indian & & 1 & & 1 & & & & 2 \\
\hline Others & 1 & & & 1 & & & & 2 \\
\hline \multicolumn{9}{|c|}{ Ethnicity (national servicemen) } \\
\hline Chinese & 8 & 2 & & & & 1 & & 11 \\
\hline Malay & 1 & & & & & & & 1 \\
\hline Indian & 5 & 1 & & & & & 1 & 7 \\
\hline
\end{tabular}


In a previous study, most Klebsiella liver abscesses in our hospital $(n=40)$ were caused by capsule type K1 $(n=16)$ and K2 $(n=8)$ strains. $^{2}$ This corresponds with the prevalence of capsule types of strains colonising the human gut in the local community based on the present study. In conclusion, about $3-8 \%$ of the local population may carry hypervirulent $K$. pneumoniae in their gastrointestinal tract. It is likely that there is a yet-unknown environmental source that leads to acquisition of these strains by ingestion.

\section{Acknowledgement}

This study was funded by a grant from the Society of Infectious Disease (Singapore) Research Development Fund.

\section{REFERENCES}

1. Turton JF, Perry C, Elgohari S, et al. PCR characterization and typing of Klebsiella pneumoniae using capsular type-specific, variable number tandem repeat and virulence gene targets. J Med Microbiol 2010;59:541-7.
2. Yeh KM, Kurup A, Siu LK, et al. Capsular serotype K1 or K2, rather than magA and $\mathrm{rmpA}$, is a major virulence determinant for Klebsiella pneumoniae liver abscess in Singapore and Taiwan. J Clin Microbiol 2007;45:466-71

Tse H Koh, ${ }^{1} M D, P h D$, Vernon Lee, ${ }^{2} M B B S, P h D$, Jeremiah $\underline{\text { Chng, }}{ }^{2}{ }_{M B B S, M P H}$, Delphine YH $\underline{\mathrm{CaO}},{ }_{B S C}^{1}$ (Hons), Boon C Khoo, ${ }_{B S C}$, Audrey HJ Tan, ${ }_{B S C}^{1}$, Peck L $\underline{\text { Tan, }},{ }^{1}$ Dip (Chemical Process Technology), Freddy JX $\underline{\mathrm{NeO}},{ }_{B S c}^{3}$, Dennis MW Heng, ${ }^{3} B S c$, Ching Ging $\underline{\mathrm{Ng}}{ }^{3}{ }_{P h D}$

\footnotetext{
${ }^{1}$ Department of Microbiology, Singapore General Hospital, Singapore

${ }^{2}$ Headquarters Medical Corps, Ministry of Defence, Singapore

${ }^{3}$ Defence Medical \& Environmental Research Institute, DSO National Laboratories, Singapore
}

Correspondence: Dr Tse Hsien Koh, Department of Microbiology, Level 7 Diagnostics Tower, 20 College Road, Academia, Singapore General Hospital, Singapore 169856.

Email: koh.tse.hsien@singhealth.com.sg 